\title{
Application of 3D-FS-SPGR imaging combined synovial fluid GGCX detection in the evaluation of knee osteoarthritis
}

\author{
Lvlin Yang ${ }^{B}$, Dongsheng Niu' ${ }^{A}$, Zhigang Bai ${ }^{\mathrm{F}}$, Jun Ma ${ }^{\mathrm{D}}$, Xichun Sun' ${ }^{\mathrm{C}}$ Yuqi Liang $^{\mathrm{C}}$, Jie Zhang ${ }^{\mathrm{A}}$ \\ Department of Orthopedics, Autonomous Region People's Hospital of Ningxia Medical University, China \\ A - research concept and design; $B$ - collection and/or assembly of data; $C$ - data analysis and interpretation; \\ $\mathrm{D}$ - writing the article; $\mathrm{E}$ - critical revision of the article; $\mathrm{F}$ - final approval of the article
}

Address for correspondence

Lvlin Yang

E-mail:gufwcbqkp4wdzuvq@sina.com

\section{Funding sources}

The study was funded by the Ningxia Natural

Science Foundation (grant No. NZ17203).

Conflict of interest

None declared

Received on 0ctober 15, 2018

Reviewed on November 12, 2018

Accepted on April 30, 2020

Published online on July 1,2020

\begin{abstract}
Background. Osteoarthritis represents a kind of chronic and degenerative joint disease characterized by articular cartilage injury and osteoproliferation. Osteoarthritis especially poses a serious threat to the elderly patients. At present, the diagnosis of osteoarthritis mainly consists of clinical examination, $X$-ray examination, magnetic resonance imaging (MRI), and arthroscopy. However, limitations and misdiagnosis are found within the single method.
\end{abstract}

Objectives. This article intends to investigate the feasibility of assessing the condition of knee osteoarthritis through quantitative analysis of cartilage using nuclear magnetic resonance 3D fast-spin spoiled gradientrecalled echo (NMR3D-FS-SPGR) imaging and y-glutamic acid carboxylase (GGCX) detection in synovial fluid.

Material and methods. A total of 60 patients with primary knee osteoarthritis were enrolled. All the patients were staged and received 3D-FS-SPGR sequence MRI scan for grading based on scan results and cartilage injury. Cartilage tissues were collected for immunohistochemistry (IHC). The GGCX in cartilage was detected using western blotting to analyze the correlation with arthritis.

Results. The condition of articular cartilage injury in arthritis patients was clearly observed using 3D-FSSPGR sequence. The expression of GGCX was decreased in 46 patients $(p<0.05)$. The expression of GGCX in synovial fluid was significantly reduced following upstaging $(p<0.05)$. The sensitivity measured using combined 3D-FS-SPGR imaging and synovial fluid GGCX detection for the evaluation of arthritis condition was significantly higher than that of the single detection method $(p<0.05)$.

Conclusions. Our data showed that the sensitivity of combined detection was obviously higher than single detection for the evaluation of arthritis. The 3D-FS-SPGR combined with synovial fluid GGCX detection could be treated as a promising strategy for arthritis evaluation.

Key words: arthritis, MRI, 3D-FS-SPGR sequence, synovial fluid, GGCX

Cite as

Yang L, Niu D, Bai Z, et al. Application of 3D-FS-SPGR imaging combined synovial fluid GGCX detection in the evaluation of knee osteoarthritis. Adv Clin Exp Med. 2020;29(6):701-706. doi:10.17219/acem/121522

DOI

10.17219/acem/121522

Copyright

Copyright by Author(s)

This is an article distributed under the terms of the

Creative Commons Attribution 3.0 Unported (CC BY 3.0)

(https://creativecommons.org/licenses/by/3.0/) 


\section{Introduction}

Osteoarthritis, as a chronic and degenerative joint disease, leads to articular cartilage damage and osteoproliferation. It dramatically impairs mobility in the elderly patients. ${ }^{1}$ Periodic evaluation and continuous monitoring of patients with arthritis are helpful and critical in the long-term management of the disease. ${ }^{2}$ Although it is possible to diagnose osteoarthritis with a clinical examination, $\mathrm{X}$-ray examination, magnetic resonanc imaging (MRI), and arthroscopy, there are still limitations and misdiagnoses which call for further improvement in the methods of diagnosis. ${ }^{3}$

Magnetic resonance imaging employs nuclear magnetic resonance (NMR) phenomena of human protons in the magnetic field to collect MR signal for diagnosis using spatial coding technology. ${ }^{4}$ Magnetic resonance imaging examination is considered as non-radiation, non-invasive and multi-faceted scanning, and characterized by high soft tissue resolution. It is advantageous in pre-treatment diagnosis and routine observation over other examination methods. ${ }^{5}$ Therefore, MRI is currently recognized as the best way to check the pathological changes of the knee. The sensitivity of MRI sequences in the diagnosis of knee joint cartilage lesions has been widely reported. For instance, it has been found that the sensitivity and specificity of 3D fast-spin spoiled gradient-recalled echo (NMR 3D-FS-SPGR) for lesions were $75-85 \%$ and $97 \%$, respectively. ${ }^{6}$ In addition, previous evidence also revealed that the sensitivity of 3D-FS-SPGR sequence in cartilage lesions was $97 \%$, and the specificity was $95 \%$ in $1.5 \mathrm{~T}$ magnetic resonance. ${ }^{7}$

A recent study indicated that $\gamma$-glutamic acid carboxylase (GGCX) reduced carboxylated matrix $\gamma$-carboxyglutamate (Gla) protein level in the cartilage of patients with osteoarthritis compared to that in the normal control group. ${ }^{8}$ In addition, it was demonstrated that GGCX concentration in articular cartilage of patients with osteoarthritis was also lower than that of normal cartilage tissue, and gradually declined as the joint injury aggravated. ${ }^{9}$ Thus, GGCX is proposed to serve to some extent as an indicator for the progression of the disease in patients with arthritis. This study intends to evaluate the value of NMR-3D-FSSPGR imaging and GGCX detection in synovial fluid in assessing the condition of knee osteoarthritis.

\section{Patients and methods}

\section{Main reagents and instruments}

Magnetic resonance imaging scan was performed with Gesigna 3.0T MRI system (GE Healthcare, Little Chalfont, UK). Immunohistochemistry and western blot antibodies were purchased from Wuhan Mitaka Biotechnology Co., Ltd (Wuhan, China). 3,3'-diaminobenzidine (DAB) was bought from Wuhan Boster Biological Engineering Co., Ltd. (Wuhan, China). Other reagents involved in this study were obtained from Sinopharm Chemical Reagent Co., Ltd. (Shanghai, China).

\section{Study participants}

A total of 60 osteoarthritis patients treated between October 2016 and October 2017 in General Hospital of Ningxia Hui Autonomous Region People's Hospital (Yinchuan, China) were enrolled, including 34 males and 26 females, with mean age of $61.7 \pm 5.1$ years (range: $57-68$ years). Another 20 healthy adults with corresponding age and gender were selected as the control group. The patients presented with repeated knee pain within the past month. X-ray (standing or weight-bearing position) showed joint space narrowing, sclerosis and/or cystic degeneration, and joint osteophyte formation. Synovial fluid (examined at least 2 times) was clear and thick red, with white blood cells (WBC) $<2000$ cells/mL. Moreover, morning stiffness was shorter than $30 \mathrm{~min}$. Bone friction sound was found during activity. All patients underwent MRI examination after admission and the synovial fluid was taken 1 week later for GGCX detection. The study was reviewed and approved by Ethics Committee of General Hospital of Ningxia Hui Autonomous Region People's Hospital and all patients participating in the study signed an informed consent.

\section{Classification criteria for articular cartilage injury}

Knee osteoarthritis was divided into 4 stages according to Kellgren-Lawrence criteria:

Stage 1: in slight discomfort in the joint after activity; joint pain and swelling after increased activity; no evidence of significant cartilage damage on X-ray and computed tomography $(\mathrm{CT})$ examination.

Stage 2: significant pain after activity and reduction after rest; X-ray showed slight change; only CT showed mild cartilage damage; isotope examination exhibited condensation in damaged joint.

Stage 3 (advanced): further osteochondral damage, resulting in joint deformity and loss of function; X-ray showed joint space narrowing, cystic lesions around the joints and articular mobile corpus.

Stage 4 (late): bone hyperplasia and cartilage exfoliation leading to complete loss of function; significant joint deformity; X-ray showed joint space narrowing, severe hyperplasia, coarse joint, and even bone collapse. ${ }^{10}$

Magnetic resonance imaging diagnostic grading was based on arthroscopic classifications. Grade 0 represents normal articular cartilage; grade I represents poorly defined cartilage structure or low focal signal within the cartilage; grade II represents mild or moderate irregularity in a cartilage surface profile, and the extent of cartilage 
defect is smaller than 50\%; grade III represents a severely irregular cartilage surface profile with more than 50\% of cartilage defects; grade IV represents full-thickness cartilage defects and subchondral bone exposure. ${ }^{11}$

\section{Inspection method}

The knees of all subjects were examined taking into account the femoral medial and lateral condyles, the tibia medial and lateral platform, the intercondylar femoral condyle, the trochlear articular surface, and patella articulation surface. The MRI scan was performed with a Gesigna 3.0T MRI system using a 3D-FS-SPGR sequence. The main parameters were the following: repetition time (TR) - $13 \mathrm{~ms}$, echo time (TE) $-3.3 \mathrm{~ms}$, thickness $-1.8 \mathrm{~mm}$, interval $-0.9 \mathrm{~mm}$, field of view (FOV) $-18 \times 16 \mathrm{~cm}$, numbers of excitations (NEX) -2 , matrix $-288 \times 256$ ppx, scan time $-348 \mathrm{~s}$. A total of $2 \mathrm{~mL}$ of right knee synovial fluid was drawn with local anesthesia of the lower extremity within 1 week after MRI scan. The synovial fluid was transferred to a centrifuge tube and centrifuged at $4,000 \mathrm{~g}$ for $10 \mathrm{~min}$ at $4^{\circ} \mathrm{C}$. The supernatant was stored at $-80^{\circ} \mathrm{C}$ for GGCX detection.

\section{Immunohistochemistry}

Articular cartilage samples were obtained from surgery and fixed in formalin. The paraffin-embedded sections were sectioned at a thickness of $3 \mu \mathrm{m}$. The paraffin sections were then dewaxed, hydrated and treated with $3 \% \mathrm{H}_{2} \mathrm{O}_{2}$ to block the endogenous peroxidase activity. After being washed with water, the sections were soaked in $0.01 \mathrm{M}$ phosphatebuffered solution (PBS; pH 7.4) for 5 min. Next, the sections were blocked in 5-10\% normal goat serum at room temperature for $10 \mathrm{~min}$ for blocking. Then, rabbit anti-human GGCX-specific primary antibody working solution was added to the sections $(1: 1,000)$ at $37^{\circ} \mathrm{C}$ for $2 \mathrm{~h}$. After being washed 3 times, the sections were incubated in biotin labeled anti-rabbit secondary antibody working solution (1:200) at $37^{\circ} \mathrm{C}$ for $10-30 \mathrm{~min}$. Next, horseradish enzymelabeled streptavidin working solution was added to the sections at $37^{\circ} \mathrm{C}$ for $10-30 \mathrm{~min}$. Finally, the sections were developed with DAB for $10 \mathrm{~min}$ and counterstained with hematoxylin for $2 \mathrm{~min}$. After dehydration and hyalinization, the sections were observed under a microscope. Results evaluation showed that GGCX was expressed in the cytoplasm. The cells were considered positive if the brown particles appeared in the cytoplasm. The positive cell ratio over $40 \%$ was treated as GGCX high expression.

\section{Western blot}

The synovial fluid samples were lysed with pre-cooled cell lysate and total protein was extracted. Then, the protein was separated using sodium dodecyl sulfate polyacrylamide gel electrophoresis (SDS-PAGE) and transferred to polyvinylidene fluoride (PVDF) membrane at $70 \mathrm{~V}$ for $3 \mathrm{~h}$. Next, the membrane was blocked in $5 \%$ skim milk at $37^{\circ} \mathrm{C}$ for $1 \mathrm{~h}$ and incubated in rabbit anti-human GGCX antibody $(1: 1,000)$ at $4^{\circ} \mathrm{C}$ overnight. After being washed with phosphate-buffered solution Tween 20 (PBST), the membrane was incubated in horseradish peroxidase (HRP)-labeled mouse anti-rabbit IgG secondary antibody (1:200) at room temperature for $1 \mathrm{~h}$. Finally, the membrane was developed in $\mathrm{DAB}$ for $10 \mathrm{~min}$ and the image was analyzed using a gel imaging analysis system (Bio-Rad, Hercules, USA).

\section{Statistical analysis}

All data analyses were performed on SPSS v. 20.0 software (IBM Corp., Armonk, USA). The qualitative data was compared using rank sum test. The enumeration data was presented as rate or percentage and compared using $X^{2}$ test. The measurement data were presented as $\mathrm{X} \pm \mathrm{s}$ and compared using t-test or one-way analysis of variance (ANOVA) with Student-Newman-Keuls (SNK) post hoc test. P-value $<0.05$ was considered statistical significance.

\section{Results}

\section{D-FS-SPGR imaging}

Knee joint damage using 3D-FS-SPGR imaging was analyzed in all the arthritic patients. The results are shown in Fig. 1. The normal articular cartilage signal was uniform and consistent without cartilage swelling. The edge was clear and smooth with no cartilage defects. 3D-FS-SPGR detection demonstrated less uniform articular cartilage signal in grade I patients. The area with increasing local
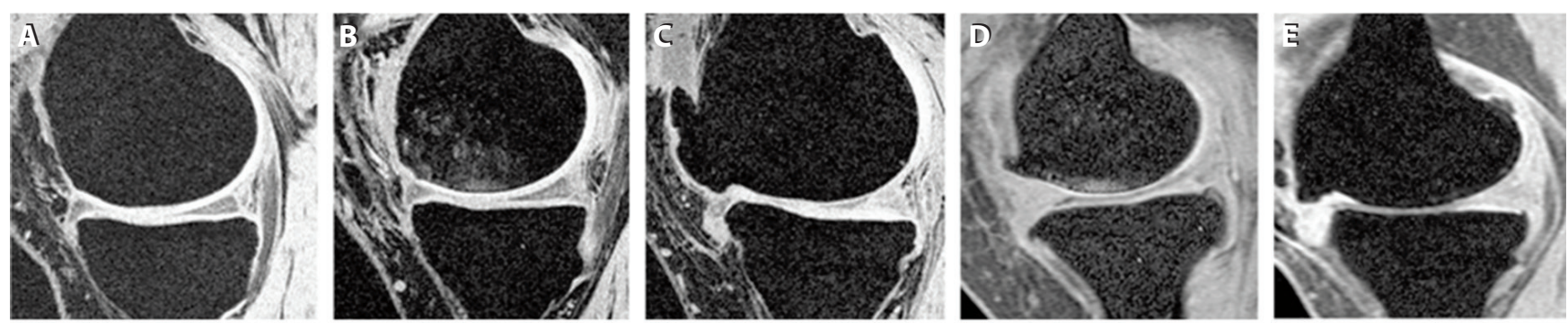

Fig. 1. 3D-FS-SPGR imaging of osteoarthritis patients: A) healthy volunteer; B) grade I; C) grade II; D) grade III; E) grade IV 

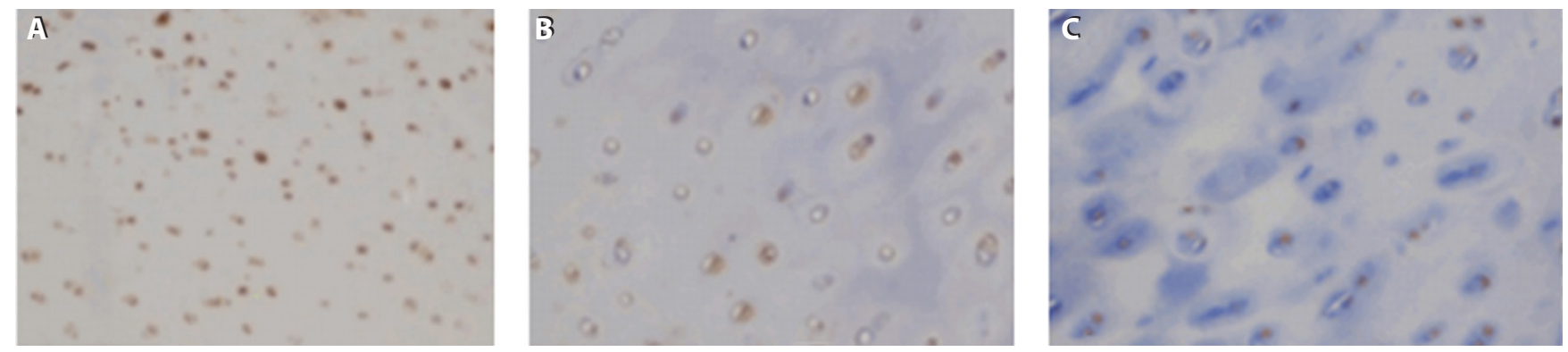

Fig. 2. GGCX expression detected using immunohistochemistry (x100). A and B. Cartilage tissue with normal GGCX expression. C. Cartilage tissue with low GGCX expression

signal could be found with no articular cartilage defect. In grade II patients, a less uniform articular cartilage signal with local thinner cartilage was observed. The defect did not reach $50 \%$ of the full thickness. Grade III patients were characterized by severe fibrosis in the anterolateral cartilage with thinner and non-uniform cartilage. The cartilage defects were more than $50 \%$ of the full-thickness, and no subchondral bone was found. In grade IV patients, the whole cartilage of the femur and tibia was lost, and the subchondral bone was exposed. High signal synovial fluid was observed within the cavity, which can be distinguished from cartilage signal.

\section{Expression of GGCX detected with immunohistochemistry}

The expression of GGCX in articular cartilage specimens was examined with immunohistochemistry. As shown in Fig. 2, significantly lower GGCX expressions in articular cartilage were presented in 46 patients compared to that of 20 healthy volunteers $(\mathrm{p}<0.01)$.

\section{Expression of GGCX in synovial fluid}

The expression of GGCX in synovial fluid was tested with western blot (Fig. 3). $\beta$-actin was selected as a loading control to calculate the relative amount of GGCX (Fig. 4). Compared with healthy volunteers, we found that the overall GGCX levels in knee articular cartilage of 39 osteoarthritis patients were significantly reduced $(\mathrm{p}<0.01)$. Moreover, GGCX expression in synovial fluid was significantly decreased following upstaging (Fig. 5).

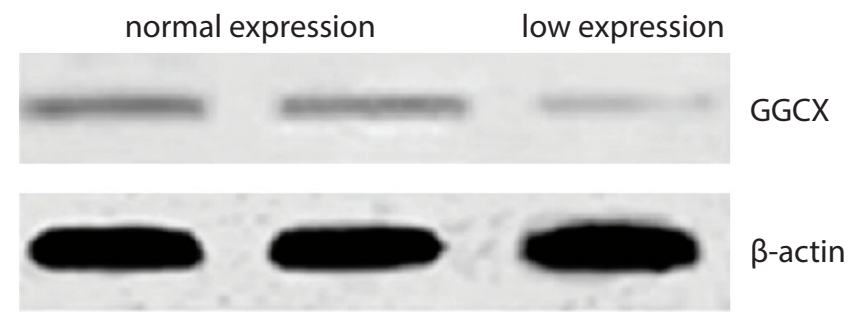

Fig. 3. GGCX expression in synovial fluid detected with western blot

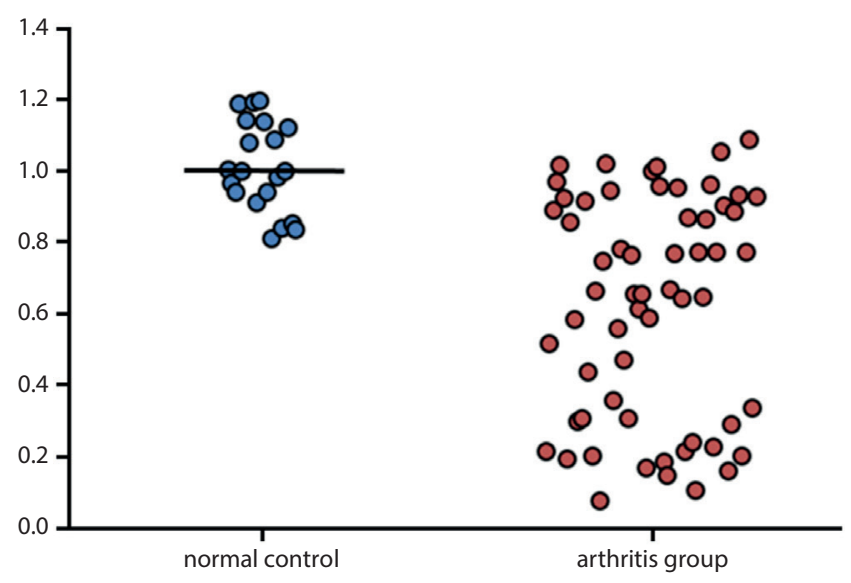

Fig. 4. GGCX expression in synovial fluid from different osteoarthritis patients

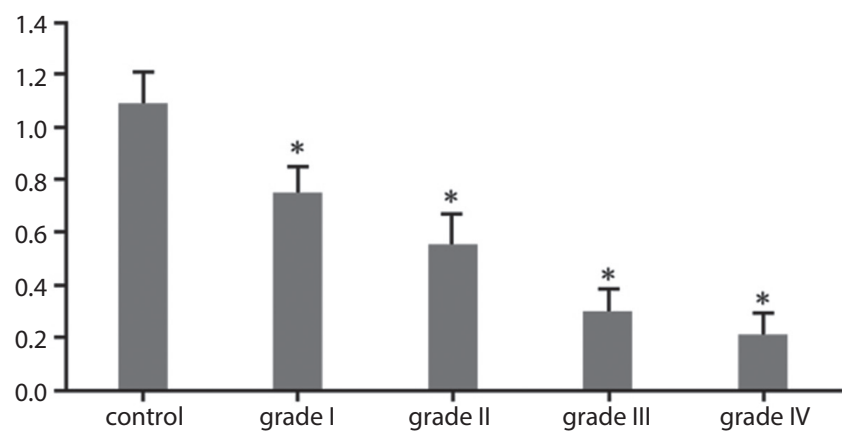

Fig. 5. GGCX expression in synovial fluid of patients with different osteoarthritis degree; ${ }^{*} p<0.05$ compared with control

\section{The relationship of MRI examination and GGCX expression with osteoarthritis grading}

The relationship between MRI examination, GGCX expression and osteoarthritis grading of all patients was listed in Table 1. The sensitivity of the 3D-FS-SPGR imaging combined synovial fluid GGCX detection for the evaluation of arthritis condition was significantly higher, nearly $100 \%$, whereas the specificity was lower than that of the single detection method $(\mathrm{p}<0.05)$. 
Table 1. The relationship of MRI examination and GGCX expression with osteoarthritis grading

\begin{tabular}{|l|c|c|c|}
\multicolumn{1}{|c|}{ Detection item } & $\begin{array}{c}\text { OA group } \\
(\mathrm{n}=60)\end{array}$ & $\begin{array}{c}\text { Non-OA group } \\
(\mathrm{n}=20)\end{array}$ & $\begin{array}{c}\text { Sensitivity } \\
{[\%]}\end{array}$ \\
\hline 3D-FS-SPGR detection & 53 & 1 & 88.3 \\
\hline GGCX detection & 36 & 4 & 60.0 \\
\hline 3D-FS-SPGR or GGCX detection & 58 & 5 & 95.0 \\
\hline 3D-FS-SPGR and GGCX detection & 41 & 0 & 70.0 \\
\hline
\end{tabular}

\section{Discussion}

Arthroscopy is used to examine osteoarthritis and evaluate cartilage injury. ${ }^{12}$ However, arthroscopy still presents drawbacks as a method for detection on the cartilage surface of the articular cavity. Its application is unable to cover the inspection of deep cartilage damage. ${ }^{12}$ Magnetic resonance imaging is currently considered the favorable method to check knee lesions. Conventional MRI sequences show the sensitivity of cartilage morphological changes. However, they normally function when the cartilage is damaged to some extent. Routine sequences neither exhibit the hierarchical representation of the cartilage nor explain the exact composition of the cartilage and the specific cause of cartilage damage. ${ }^{13}$ This study thus aims to evaluate the progression of arthritis through 3D-FS-SPGR sequence imaging in MRI and synovial fluid GGCX detection.

Knee cartilage contains water, type II collagen and proteoglycan polymer, ${ }^{14}$ which can be divided into 4 layers from inside to outside: calcified layer, radioactive layer, transition layer, and tangential layer. ${ }^{15}$ The working principle of MRI is to generate different signal changes to the structure in cartilage matrix through different magnetic fields. Magnetic resonance imaging can accurately reflect the degree of pathophysiology of articular cartilage development. Rubenstein et al. found that the spin-echo (SE) sequence and the 3D-FSSPGR sequence presented the normal articular cartilage as 3 layers, including the top phase as the tangential layer, the transition layer and the upper part of the radioactive layer; the middle layer as major of the radioactive layer; and the deep layer as the lower part of the radioactive layer. ${ }^{16}$ Combined with arthroscopic classification of cartilage injury, the damage occurred in the cartilage tangential and transitional layer in grade I-II degeneration (mild cartilage damage). On the other hand, the lesion reaches the radioactive layer at the stage of the moderate and severe cartilage injury. ${ }^{17}$ In the present study, we found that articular cartilage damage and degree can be clearly demonstrated using 3D-FS-SPGR sequence imaging.

The development of osteoarthritis is closely related to the formation of calcium microcrystals, including calcium phosphate (BCP) microcrystals and calcium pyrophosphate dihydrate (CPPD) crystals. Matrix Gla protein
(MGP) is not only expressed in articular cartilage, but also shows a close relationship with cartilage calcification and calcium microcrystal formation. ${ }^{18}$ Uncarboxylated MGP (ucMGP) content was significantly increased in the serum and synovial fluid of arthritis patients. The MGP contains 5 vitamin K-dependent amino acid GGCX (Gla) residues, while Gla shows high affinity to calcium and hydroxyapatite, but can only be activated through GGCX carboxylation. ${ }^{19}$ It has been demonstrated that GGCX expression in primary osteoarthritis cartilage was significantly lower than in normal cartilage and gradually decreased according to the degree of aggravation. The GGCX levels in synovial fluid of the primary knee osteoarthritis group were significantly lower than in the control group, which is consistent with our data. ${ }^{20}$ In addition, it was also found that the accuracy of the knee MRI 3D-FS-SPGR sequence in assessing cartilage lesions was $95.0 \% .{ }^{21}$ Previous findings indicated that MRI imaging, 3D-FS-SPGR sequence can accurately reflect the changes of articular cartilage morphology and thickness, and thus can accurately evaluate the degree of articular cartilage degeneration. ${ }^{22}$ In this study, the sensitivity of the combination of 3D-FS-SPGR and synovial fluid GGCX detection reached even $96.67 \%$, which was significantly higher than the sensitivity of a single measurement. However, limitations in our study still exist. The investigation regarding the mechanism ought to be further performed in order to formulate a broader understanding of the diagnosis and therapy of osteoarthritis.

\section{Conclusions}

Our data demonstrated that the sensitivity of combined detection was significantly improved compared with single detection for the evaluation of arthritis. This strategy may provide practical basis for the prevention and treatment of arthritis.

\section{References}

1. Bijlsma JW, Berenbaum F, Lafeber FP. Osteoarthritis: An update with relevance for clinical practice. Lancet. 2011;377(9783):2115-2126.

2. McAlindon TE, Bannuru RR, Sullivan MC, et al. Response to Letter to the Editor entitled "Comments on OARSI guidelines for the nonsurgical management of knee osteoarthritis". Osteoarthr Cartil. 2014; 22(6):890-891.

3. Loeser RF, Goldring SR, Scanzello CR, Goldring MB. Osteoarthritis: A disease of the joint as an organ. Arthritis Rheum. 2012;64(6):1697-1707. 
4. Giedd JN, Rapoport JL. Structural MRI of pediatric brain development: What have we learned and where are we going? Neuron. 2010; 67(5):728-734.

5. Levman J, MacDonald P, Lim AR, Forgeron C, Takahashi E. A pediatric structural MRI analysis of healthy brain development from newborns to young adults. Hum Brain Mapp. 2017;38(12):5931-5942.

6. Li X, Benjamin Ma C, Link TM, et al. In vivo T(1rho) and T(2) mapping of articular cartilage in osteoarthritis of the knee using 3 TMRI. Osteoarthr Cartil. 2007;15(7):789-797.

7. Lee JB, Cha JG, Lee MH, Lee YK, Lee EH, Jeon CH. Usefulness of IDEAL T2-weighted FSE and SPGR imaging in reducing metallic artifacts in the postoperative ankles with metallic hardware. Skeletal Radiol. 2013;42(2):239-247.

8. Cavaco S, Viegas CS, Rafael MS, et al. Gla-rich protein is involved in the cross-talk between calcification and inflammation in osteoarthritis. Cell Mol Life Sci. 2016;73(5):1051-1065.

9. Rafael MS, Cavaco S, Viegas CS, et al. Insights into the association of Gla-rich protein and osteoarthritis, novel splice variants and gamma-carboxylation status. Mol Nutr Food Res. 2014;58(8):1636-1646.

10. Schiphof D, Boers M, Bierma-Zeinstra SM. Differences in descriptions of Kellgren and Lawrence grades of knee osteoarthritis. Ann Rheum Dis. 2008;67(7):1034-1036.

11. Azuma T, Nakai $R$, Takizawa $O$, Tsutsumi S. In vivo structural analysis of articular cartilage using diffusion tensor magnetic resonance imaging. Magn Reson Imaging. 2009;27(9):1242-1248.

12. Goldring MB, Goldring SR. Articular cartilage and subchondral bone in the pathogenesis of osteoarthritis. Ann N Y Acad Sci. 2010;1192: 230-237.

13. Hunter DJ, Arden N, Conaghan PG, et al. Definition of osteoarthritis on MRI: Results of a Delphi exercise. Osteoarthr Cartil. 2011;19(8): 963-969.
14. Potter HG, Jain SK, Ma Y, Black BR, Fung S, Lyman S. Cartilage injury after acute, isolated anterior cruciate ligament tear: Immediate and longitudinal effect with clinical/MRI follow-up. Am J Sports Med. 2012; 40(2):276-285.

15. Mosher TJ, Liu Y, Torok CM. Functional cartilage MRI T2 mapping: Evaluating the effect of age and training on knee cartilage response to running. Osteoarthr Cartil. 2010;18(3):358-364.

16. Li X, Yu C, Wu H, et al. Prospective comparison of 3D FIESTA versus fat-suppressed 3D SPGR MRI in evaluating knee cartilage lesions. Clin Radiol. 2009;64(10):1000-1008.

17. Souza RB, Stehling C, Wyman BT, et al. The effects of acute loading on T1rho and T2 relaxation times of tibiofemoral articular cartilage. Osteoarthr Cartil. 2010;18(12):1557-1563.

18. Ea HK, Nguyen C, Bazin D, et al. Articular cartilage calcification in osteoarthritis: Insights into crystal-induced stress. Arthritis Rheum. 2011;63(1):10-18.

19. Cranenburg EC, Brandenburg VM, Vermeer C, et al. Uncarboxylated matrix Gla protein (UCMGP) is associated with coronary artery calcification in haemodialysis patients. Thromb Haemost. 2009;101(2): 359-366.

20. De Vilder EY, Debacker J, Vanakker OM. GGCX-associated phenotypes: An overview in search of genotype-phenotype correlations. Int J Mol Sci. 2017;18(2):240.

21. Yoshioka H, Alley $M$, Steines $D$, et al. Imaging of the articular cartilage in osteoarthritis of the knee joint: 3D spatial-spectral spoiled gradient-echo vs fat-suppressed 3D spoiled gradient-echo MR imaging. J Magn Reson Imaging. 2003;18(1):66-71.

22. Wenger A, Englund M, Wirth W, Hudelmaier M, Kwoh K, Eckstein F; OAl Investigators. Relationship of 3D meniscal morphology and position with knee pain in subjects with knee osteoarthritis: A pilot study. Eur Radiol. 2012;22(1):211-220.

23. Fan MP, Si M, Li BJ, et al. Cell therapy of a knee osteoarthritis rat model using precartilaginous stem cells. Eur Rev Med Pharmacol Sci. 2018;22(7):2119-2125. 